\title{
Delayed presentation of inflammatory breast carcinoma during the COVID-19 pandemic
}

\author{
Daniele Bernardi · Emanuele Asti · Giulia Bonavina · Alberto Luporini · Claudio Clemente · Luigi Bonavina $\mathbb{D}$
}

Received: 1 May 2021 / Accepted: 4 May 2021 / Published online: 15 June 2021

(c) Springer-Verlag GmbH Austria, part of Springer Nature 2021

\begin{abstract}
Summary
Background Breast cancer may present with distinct cutaneous manifestations that may be paraneoplastic or secondary to direct skin infiltration, distant skin metastases, or dermal lymphatic tumor embolization (inflammatory breast carcinoma).

Case report A 51-year-old Asian woman visited the emergency care department during the outbreak of COVID-19 in Northern Italy. About 6 months before, she had noted the onset of right breast swelling accompanied by skin redness and itching. She never consulted a physician, and, over time, the local skin condition progressed to a large scaly plaque covering the entire breast surface including the nipple. At presentation, abduction of the right upper limb was impaired due to severe shoulder pain. CT scan showed the presence of bilateral breast masses with necrotic and colliquative features, and multiple skeletal, nodal, pulmonary, and brain images suggestive of metastases. An ultrasound-guided core biopsy of the con-
\end{abstract}

D. Bernardi, MD $\cdot$ E. Asti, MD

Department of Biomedical Sciences for Health, Division of General Surgery, University of Milan, Milan, Italy

\section{G. Bonavina, MD}

Department of Gynecology, Vita e Salute University,

Ospedale San Raffaele, Milan, Italy

\section{A. Luporini, $\mathrm{MD}$}

Medical Oncology Unit, IRCCS Policlinico San Donato, Milan, Italy

\section{Clemente, MD}

Pathology and Cytopathology Unit, IRCCS Policlinico San Donato, Milan, Italy

Prof. L. Bonavina, MD, FACS (ه)

IRCCS Policlinico San Donato, Piazza Edmondo Malan 1, San Donato Milanese, 20097 Milan, Italy

luigi.bonavina@unimi.it tralateral breast showed grade 2 non-special type infiltrating carcinoma. The patient was referred to the breast oncology unit and is currently being treated with aromatase inhibitors and chemotherapy.

Conclusion The COVID-19 pandemic has disrupted the entire spectrum of oncological care including breast cancer. Hopefully, telemedicine will contribute to increase patients' confidence and will provide earlier diagnosis and treatment while minimizing the risk of contagion.

Keywords Breast cancer - Breast cancer screening Skin metastases - Paraneoplastic dermatosis - Paget disease

\section{Main novel aspects}

- The COVID-19 pandemic has disrupted the health care system worldwide, causing delayed oncological care.

- An Asian woman presented with late-stage inflammatory breast carcinoma with impressive skin changes.

\section{Introduction}

Breast carcinoma is the most common malignancy leading to skin metastasis in women. The overall reported rate is as high as $24 \%$, but only $3 \%$ of patients present with cutaneous metastasis as the initial sign of the disease. Skin nodules from primary breast cancer are the most common clinical presentation and represent the most common metastases diagnosed by dermatologists [1]. Tumor cells can reach the skin through direct invasion or through hematogenous or lymphatic spread. Inflammatory breast carcinoma (IBC) is the most aggressive form of breast cancer, accounts for less than $5 \%$ of cases, and dermal 
Fig. 1 Extensive skin changes overlying the right breast $(\mathbf{a}, \mathbf{b})$. Details of the scaly patches (c)
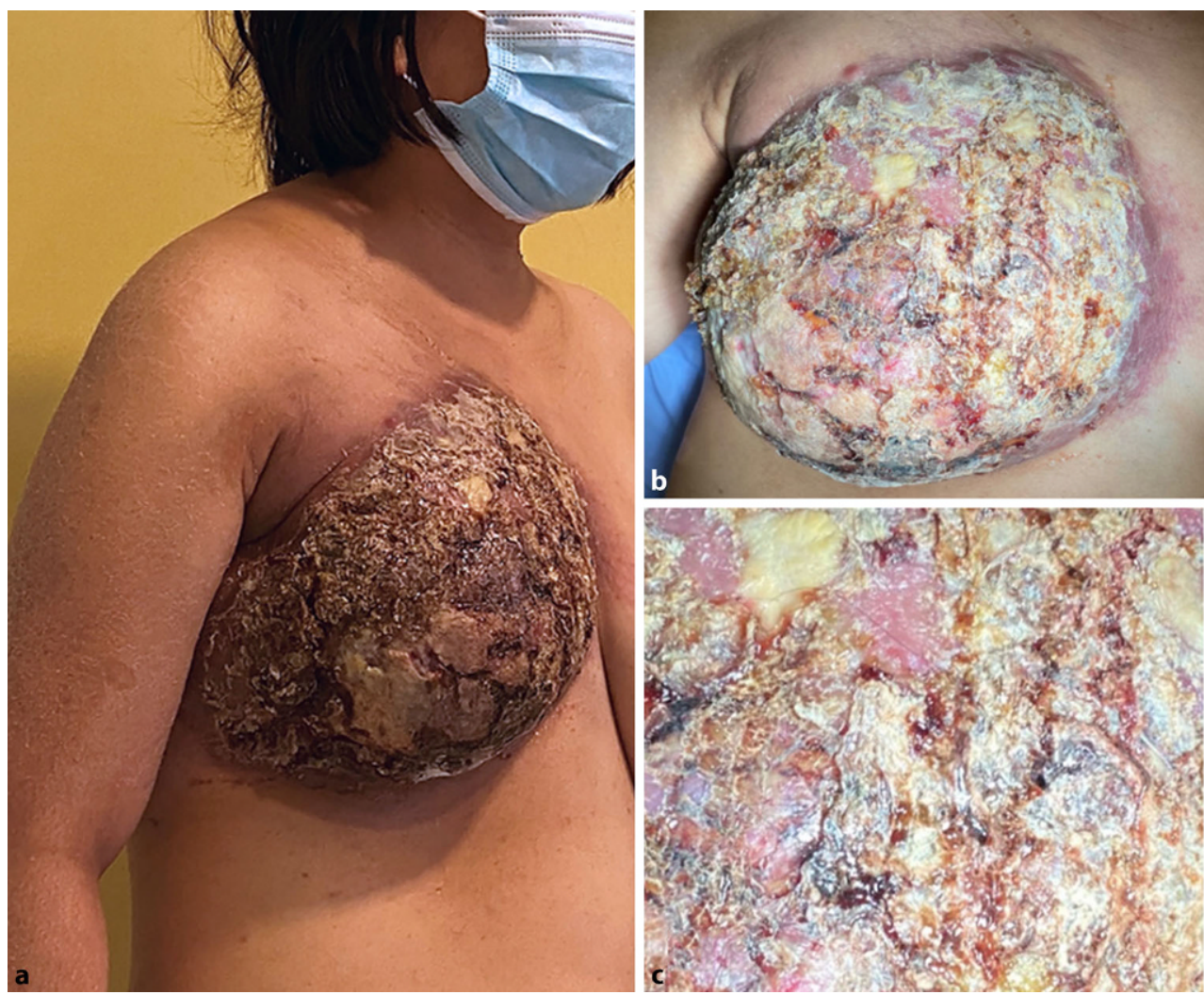

lymphatic involvement represents the essential feature. Because of its rarity, IBC is often misdiagnosed as mastitis [2]. On the other hand, a variety of paraneoplastic dermatoses may lead to incidental diagnosis of associated breast cancer [3]. We report the case of metastatic breast carcinoma with local cutaneous involvement that was diagnosed during the first peak of COVID-19 outbreak in Northern Italy.

\section{Case report}

A 51-year-old Asian woman presented to the emergency department on May 6, 2020, during the lockdown for the COVID-19 outbreak. She was nulliparous, a working immigrant, with low educational level, living with her husband. About 6 months previously, she had noted the onset of right breast swelling with skin redness and itching. This condition progressively worsened, causing major discomfort and associated shoulder pain with reduced mobility of the shoulder and right arm. The patient did not seek any medical consultation, as she did not trust her general practitioner and was ashamed and feared of visiting hospitals during the COVID-19 pandemic. At physical examination, the entire skin surface of the right breast including the nipple was replaced by a large scaly plaque with areas of yellow-brown crusting alternated with the presence of fibrin and serous secretions. The edges of this large skin lesion were erythematous and edematous, with subcutaneous swelling extended from the right clavicle to the ipsilateral flank (Fig. 1). Hard consistency tissue was palpable in the left breast. Blood analysis showed anemia $(\mathrm{Hb} 8.1 \mathrm{~g} / \mathrm{dL})$, leukocytosis $\left(21.30 \times 10^{\wedge} 3 \mu \mathrm{L}\right)$, and increased C-reactive protein $(13.2 \mathrm{mg} / \mathrm{dL})$. Multisensitive Staphylococcus aureus was isolated from two different skin swabs, and therapy with daptomycin was initiated. The SARS-CoV-2 polymerase chain reaction test was negative. A chest CT scan showed the presence of bilateral breast masses, the largest in the right breast $(98 \times 52 \mathrm{~mm})$ with necrotic and colliquative features, and enlarged nodes visible in the right axilla. In addition, there were multiple bone metastases involving the sternum and, on the right hemisoma, the first rib, scapula, and humerus (Fig. 2a,b); a right pleural effusion; and left pulmonary metastases (Fig. 2b,c). A head CT scan showed multiple metastatic brain lesions and skull erosion (Fig. 3a,b).

An ultrasound-guided core biopsy of the left breast was performed, and histology showed a non-special type (NST) infiltrating carcinoma, grade 2 according to the Elston-Ellis modification of the original Scarff-Bloom-Richardson (SBR) system ([4]; Fig. 4a, b, c). Estrogen receptors (ER) were positive (Fig. 4d), while progesterone receptors (PR) and human epidermal growth factor receptor 2 (c-erbB2) were negative (Fig. 4e); Ki-67 immunohistochemistry was positive (80\%; Fig. 4f).

The patient was referred to the breast oncology unit with a presumptive diagnosis of IBC, and treatment with non-steroidal aromatase inhibitors (letrozole $2.5 \mathrm{mg}$ per oral/daily) and chemotherapy with cy- 

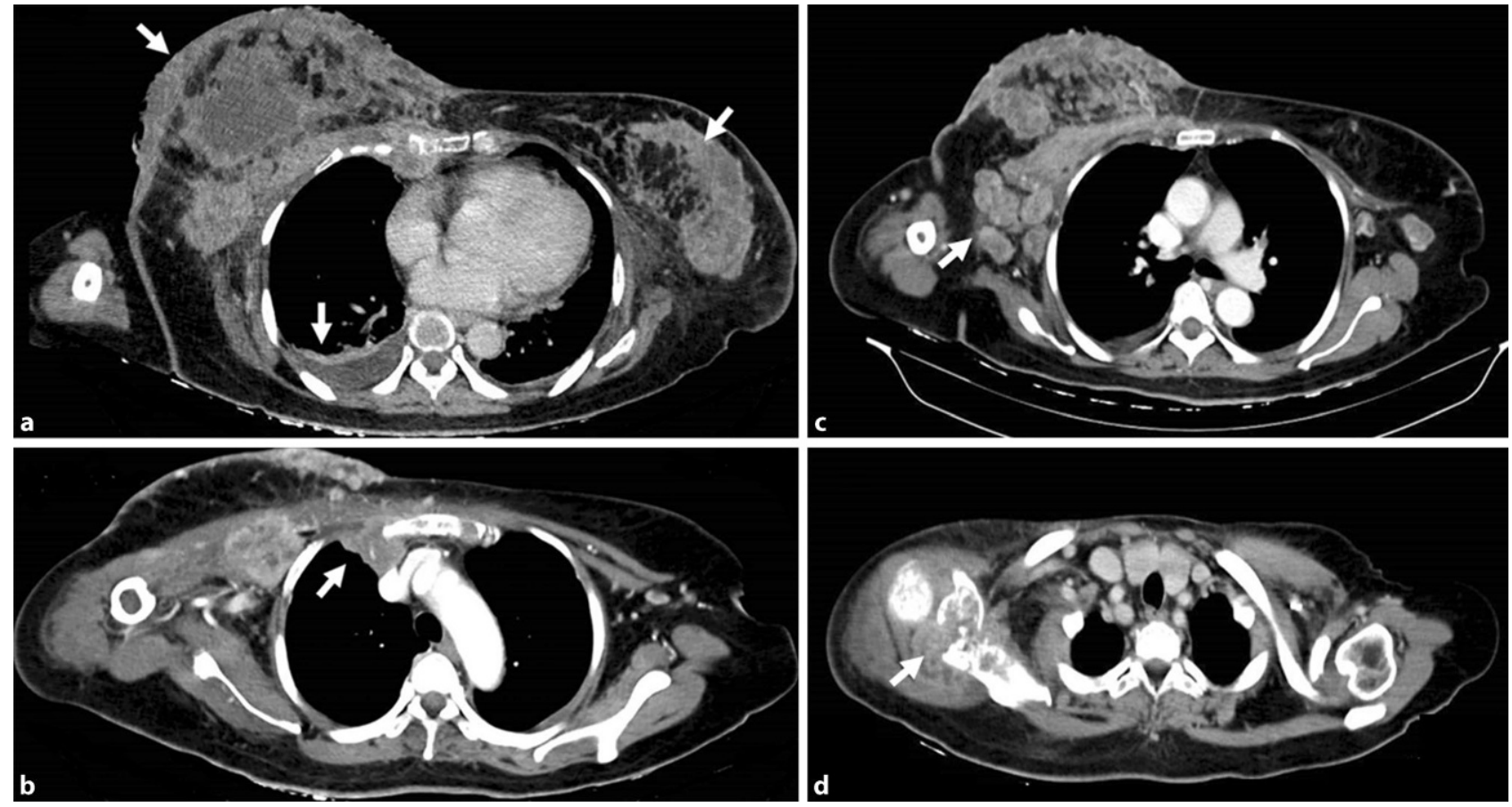

Fig. 2 Computed tomography scan showing bilateral breast masses, the largest in the right breast $(98 \times 52 \mathrm{~mm})$ with necrotic and colliquative features (a); sternum and rib in-

vasion (b); enlargement of axillary nodes (c); scapula and humerus head invasion (d). White arrows indicate the sites of metastasis

Fig. 3 Head CT scan showing skull erosion and multiple brain metastases. White arrows indicate the sites of metastasis
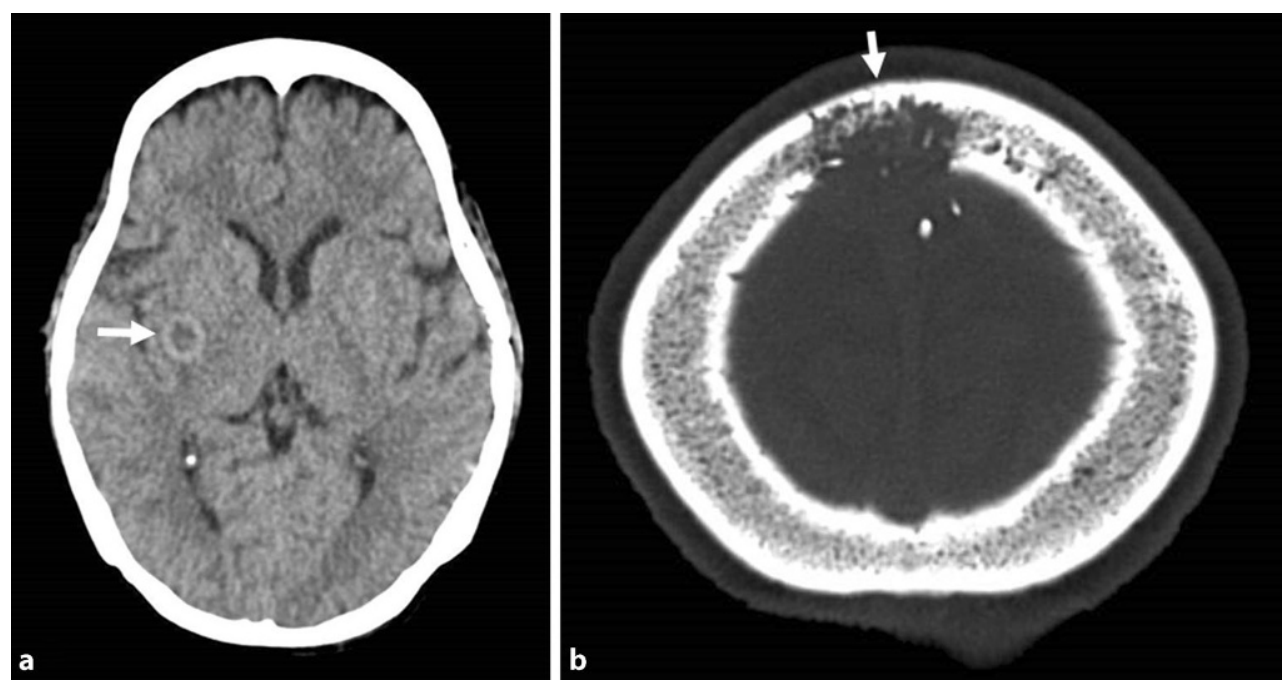

clin-dependent kinase CDK 4/6 inhibitors (palbociclib $125 \mathrm{mg}$ per oral/daily) was initiated.

\section{Discussion}

The COVID-19 outbreak has caused severe disruption in national health care systems, including suspended screening programs and delayed breast cancer diagnosis and treatment. It has been reported that delays up to 3-6 months in breast cancer diagnosis are associated with lower survival $[5,6]$. The diagnostic delay may be attributed to patients' refractoriness in accessing medical care and/or system delay in providing medical consultation and a definitive diagnosis. Although the oncological impact of the COVID19 pandemic has yet to be fully analyzed, it is likely that an increased rate of more advanced breast cancers will be discovered over time and major efforts are required to reorganize screening programs and to address a rising rate of elective cancer operations [7, 8]. Nonetheless, despite the fact that the workload for breast cancer has been high during the pandemic peak in Northern Italy, hubs have allowed the continuation of oncologic treatments, especially radiother- 


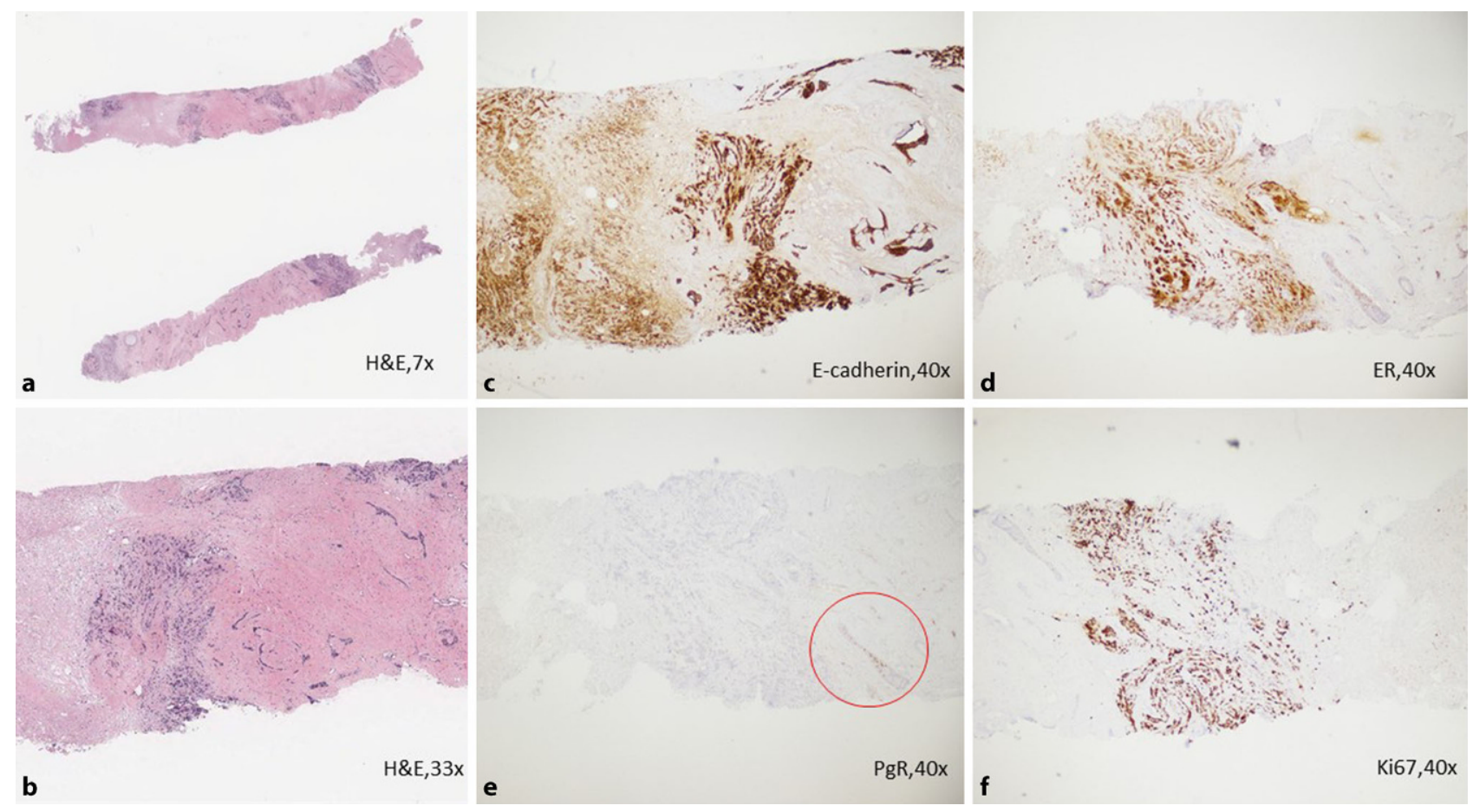

Fig. 4 a, b Non-special type (NST) infiltrating carcinoma of the left breast. a Core biopsy. $\mathbf{b}$ In the central area infiltrating carcinoma, at left necrosis and at right fibroadenomatoid mastopathy. c Immunohistochemical anti-cadherin antibody positivity of the central neoplastic cells and in the ductal cells of fibroadenomatoid mastopathy; a cross-chromogen reactivity of the necrotic area. d Immunohistochemical positivity to estrogen anti-receptor serum with weak positivity even in

apy, while mitigating the burden on frontline COVID19 hospitals [9].

In our patient, the impressive clinical presentation with extensive skin changes covering the entire right breast was likely due to permeation of skin lymphatics by metastatic emboli similar to what is seen in late-stage inflammatory breast cancer (IBC) [2]. Unfortunately, no skin punch biopsy nor core biopsy of the underlying parenchymal lesion was performed. Paget's disease and a paraneoplastic ichthyosis-like dermatosis were reasonably excluded because skin changes were limited to the breast surface and involvement of the nipple occurred late in the clinical course of the disease. It is estimated that $1-3 \%$ of all breast cancers present with the features of IBC involving the dermal lymphatic vessels, and about $40 \%$ of these patients have stage IV disease with synchronous distant metastases [10]. Inflammatory breast cancer is characterized by aggressive clinical behavior with rapid disease progression, and is a distinct biological entity rather than a subtype of locally advanced breast cancer. Among the risk factors for IBC are African ethnicity, high body mass index, and younger age at disease onset. A multimodal approach can significantly improve patients' outcomes if started early in the course of the disease, and systemic therapy should be followed by radiotherapy and/or mastec- the ductal cells of fibroadenomatoid mastopathy (positive internal control). e Immunohistochemical negativity to the progesterone serum with focal (red circle) positivity in the ductal cells of fibroadenomatoid mastopathy (positive internal control). f Ki67 immunohistochemical positive stain in the central area of carcinoma and negative ductal cells in fibroadenomatoid mastopathy and in the necrotic area

tomy. Unfortunately, IBC is frequently misdiagnosed as mastitis and patients may receive several courses of antibiotics before receiving the correct diagnosis [2]. Magnetic resonance imaging is a highly accurate and important diagnostic and staging tool in patients with potential IBC $[11,12]$. More than half of patients exhibit an estrogen receptor-negative tumor, and about one third of these patients present with triplenegative tumors [2]. Of note, the diffuse pattern of ductal breast carcinoma of no special type (NST) [13] is largely underrecognized and rarely reported in the literature. Overall, based on morphology and E-cadherin expression, about $20 \%$ of patients with diffuse-type tumors are NST and have a poor survival irrespective of the molecular phenotype [14, 15].

\section{Conclusion}

During the COVID-19 pandemic, the reduced ability to offer face-to-face medical consultation has been switched to teleconsultation in many countries. Hopefully, the rapid diffusion of telemedicine driven by the pandemic will contribute to increase patients' confidence and limit the collateral damage secondary to health care prioritization and resources reallocation, while minimizing the risk of contagion [5]. This may provide reassurance and trust to many women, 
and may offer to others the chance to be diagnosed and treated at an earlier disease stage.

Acknowledgements This work was supported by AIRES ( $A s$ sociazione Italiana Ricerca ESofago)

Funding There was no funding for this study.

Author Contribution Daniele Bernardi collected the patient's data and wrote the first draft of the manuscript; Emanuele Asti, Giulia Bonavina, and Alberto Luporini collected the literature and revised the manuscript for important intellectual content; Claudio Clemente collected the figures and wrote the figure legends; Luigi Bonavina revised the final draft of the manuscript. All authors approved the final version of the manuscript.

\section{Declarations}

Conflict of interest D. Bernardi, E. Asti, G. Bonavina, A. Luporini, C. Clemente, and L. Bonavina declare that they have no competing interests.

Ethical standards This study was carried out within an appropriate ethical framework and according to internationally accepted standards for clinical practice. Patient informed consent was acquired for the diagnostic and therapeutic procedures. Written informed consent was obtained from the patient for publication of this case report and any accompanying images.

\section{References}

1. Tan AR. Cutaneous manifestations of breast cancer. Semin Oncol. 2016;43:331-4.

2. Robertson FM, Bondy M, Yang W, et al. Inflammatory breast cancer. The disease, the biology, the treatment. CACancer J Clin. 2010;60:351-75.

3. Song YJ, Wu YF, Fan T. Dermatosis as the initial manifestation of malignant breast tumors: retrospective analysis of 4 cases. Breast Care. 2010;5:174-6.
4. Lakhani SR, Ellis IO, Schnitt SJ, et al., editors. WHO classification of tumours of the breast. 4th ed. WHO classification of tumours series, Vol. 4. Lyon: International Agency for Research on Cancer; 2012. http:// publications. iarc.fr/14.

5. Richards MA, Westcombe AM, Love SB, Littlejohns P, Ramirez AJ. Influence of delay on survival in patients with breast cancer: a systematic review. Lancet. 1999;353:1119-26.

6. Sud A, Jones ME, Broggio J, et al. Collateral damage: the impact on outcomes from cancer surgery of the COVID-19 pandemic. Ann Oncol. 2020;31(8):1065-74.

7. Richards M, Anderson M, Carter P, Ebert BL, Mossialos E. The impact of the Covid-19 pandemic on cancer care. Nat Cancer. 2020;1:565-7.

8. Vanni G, Pellicciaro M, Materazzo M, Palombi L, Buonomo O. Breast cancer diagnosis in coronavirus era: alert from Italy. Front Oncol. 2020;10:938.

9. Leonardi MC, Montagna E, Galimberti V, et al. Breast adjuvant radiotherapy amid the COVID-19 crisis in a hub cancer center, Lombardy, Italy. Breast Care. 2020; https:// doi.org/10.1159/000513227.

10. Van Huden DJP, van Maaren MC, Strobbe LJA, et al. Metastatic behavior and overall survival according to breast cancer subtypes in stage IV inflammatory breast cancer. Breast Cancer Res. 2019;21:113.

11. Kalli S, Freer PE, Rafferty EA. Lesions of the skin and superficial tissue at breast MR imaging. Radiographics. 2010;30:1981-1913.

12. Le-Petross HT, Cristofanilli M, Carkaci S, et al. MRI features of inflammatory breast cancer. AJR. 2011;197:W769-W76.

13. Cheatle GL. Clinical remarks on the early recognition of cancer of the breast. BMJ. 1906;1:1205-10.

14. Pekar G, Hofmeyer S, Tabar L, et al. Multifocal breast cancer documented in large-format histology sections. Cancer. 2013;119:1132-9.

15. Tot T. Diffuse invasive breast carcinoma of no special type. Virchows Arch. 2016;468:199-206.

Publisher's Note Springer Nature remains neutral with regard to jurisdictional claims in published maps and institutional affiliations. 